\title{
Longer sleep duration may negatively affect renal function
}

\author{
Mohsen Mazidi ${ }^{1}$ Niloofar Shekoohi ${ }^{2} \cdot$ Niki Katsiki $^{3} \cdot$ Maciej Banach $^{4,5,6}$ D
}

Received: 17 August 2020 / Accepted: 24 August 2020 / Published online: 24 September 2020

(c) The Author(s) 2020

\begin{abstract}
Background Observational studies evaluating the link between sleep duration and kidney function reported controversial results. In the present study, Mendelian randomization analysis was applied to obtain unconfounded estimates of the casual association of genetically determined sleep duration with estimated glomerular filtration rate and the risk of chronic kidney disease.

Methods Data from the largest genome-wide association studies on self-reported and accelerometer-derived sleep duration, estimated glomerular filtration rate and chronic kidney disease were analysed in total, as well as separately in diabetic and non-diabetic individuals. Inverse variance weighted (IVW) method, weighted median-based method, MR-Egger and MR-Pleiotropy RESidual Sum and Outlier (MR-PRESSO) were applied, as well as the leave-one-out method to rule out the impact of single single-nucleotide polymorphism.

Results Individuals with genetically longer self-reported sleep duration had a higher chronic kidney disease risk (IVW: $\beta=0.358, p=0.047$ ). Furthermore, in non-diabetics, longer self-reported sleep duration was negatively associated with estimated glomerular filtration rate (IVW: $\beta=-0.024, p=0.020$ ). Similarly, accelerometer-derived sleep duration was negatively related to estimated glomerular filtration rate in the total population (IVW: $\beta=-0.019, p=0.047$ ) and then ondiabetic individuals. No significant association was found between self-reported sleep duration and estimated glomerular filtration rate in the whole population and type-2 diabetes mellitus patients. None of the estimated associations was subjected to a significant level of heterogeneity. MR-PRESSO analysis did not show any chance of outliers for all estimates. The pleiotropy test also indicated low chance of pleiotropy. The leave-one-out method demonstrated that the links were not driven by single-nucleotide polymorphisms.

Conclusions For the first time, the present study shed a light on the potential harmful effects of longer sleep duration (measured both objectively and subjectively) on kidney function. This finding was observed in the total population and in nondiabetic individuals, but not in those with diabetes. Further research is needed to elucidate the links between sleep duration, estimated glomerular filtration rate and the risk of chronic kidney disease.
\end{abstract}

Keywords Mendelian randomization · Sleep duration Chronic kidney disease $\cdot$ Estimated glomerular filtration rate

Mohsen Mazidi

mohsen.mazidi@kcl.ac.uk

$\triangle$ Maciej Banach

maciejbanach77@gmail.com

1 Department of Twin Research and Genetic Epidemiology, King's College London, St Thomas' Campus, Lambeth Palace Road, London SE1 7EH, UK

2 Department of Cellular and Molecular Nutrition, School of Nutritional Sciences and Dietetics, University of Medical Sciences, Tehran, Iran
3 First Department of Internal Medicine, Center for Diabetes, Metabolism and Endocrinology, AHEPA University Hospital, Thessaloniki, Greece

4 Department of Hypertension, Chair of Nephrology and Hypertension, WAM University Hospital, Medical University of Lodz, Zeromskiego 113, 90-549 Lodz, Poland

5 Polish Mother's Memorial Hospital Research Institute (PMMHRI), Lodz, Poland

6 Cardiovascular Research Centre, University of Zielona Gora, Zielona Gora, Poland 


\section{Introduction}

Adequate sleep is crucial for the regulation of body metabolism and various physiological functions [1]. Observational studies have previously reported that sleeping patterns are closely linked to morbidity and mortality [2, 3], as well as with chronic metabolic disorders, including type 2 diabetes mellitus (T2DM), respiratory diseases, hypertension and obesity [4-8]. Considering the harmful effects of short sleep duration on glucose and insulin homeostasis, it has been suggested that sleep duration may also influence renal function [9-15]. Furthermore, animal studies showed that the majority of kidney physiological mechanisms (such as the regulation of the renin-angiotensin system, sodium reabsorption, renal blood flow, glomerular filtration, and filtration fraction) are related to the diurnal rhythm [16].

Although the effects of sleep duration on several diseases have been studied, its association with chronic kidney disease (CKD) has not been widely evaluated and the available results are still controversial [9-15]. In this context, a higher risk of CKD has been reported mainly for individuals with shorter sleep duration [9]. In a prospective cohort of 4238 participants from the Nurse Health Study over an 11-year period, shorter sleep duration was associated with a rapid decline in estimated glomerular filtration rate (eGFR) [11]. Moreover, in another prospective study of 6834 Japanese individuals, short sleep duration ( $\leq 6 \mathrm{~h}$ per night) was a predictor of proteinuria [12]. On the other hand, a population-based study of Malay and Indian adults with diabetes showed that long sleep duration $(>8 \mathrm{~h}$ per night) was linked to lower eGFR [13]. In the same study, both long ( $>8 \mathrm{~h})$ and very short $(<5 \mathrm{~h})$ duration of sleep were associated with albuminuria [13]. Of note, a previous meta-analysis found a direct association of short sleep duration with proteinuria (but not with the risk of CKD) [10]. In another study, a significant association between short sleep duration and higher risk for diabetic nephropathy has been reported [15]. Ohkuma et al. reported that both short and long duration of sleep were related to a higher urinary albumin-to-creatinine ratio (UACR) and albuminuria, whereas long sleep duration was associated with a lower eGFR [14]. However, it should be noted that epidemiological studies provide poor estimates of the role of exposure (sleep duration) on disease development, and thus these studies are prone to bias.

In the present study, we performed Mendelian randomization (MR) [17] analysis to circumvent the limitations of the epidemiological studies (i.e. residual bias, confounding factors and reverse causation), aiming at assessing the associations between sleep duration, eGFR and the risk of CKD. We defined sleep duration by both subjective (i.e. selfreported) and objective (derived by accelerometer) measurements [18].

\section{Methods}

\section{Study design}

A two-sample MR study design was used. Summary statistics were obtained from the largest genome wide association studies (GWAS) on sleep duration and interested outcomes. We applied methods to estimate the unbiased effect of sleep duration on eGFR and the risk of CKD (defined as $\mathrm{eGFR}<60 \mathrm{ml} / \mathrm{min} / 1.73 \mathrm{~m}^{2}$ ).

\section{Genetic instruments for sleep duration}

Genotyping, quality control, and imputation procedures in the UK Biobank (UKB) are described elsewhere [19]. From the largest GWAS, 78 SNPs were identified to be associated with sleep duration (self-reported) among individuals of European ancestry $(n=446,118)$ (Table 1). More information can be found elsewhere [20].

We applied on the GWAS performed in the UK Biobank for the accelerometer-driven sleep duration data (an hour increase of nocturnal sleep duration) to be compared with causal estimates obtained using genetic variants associated with self-reported sleep duration. Further details on this process can be found elsewhere [21]. If a single-nucleotide polymorphism (SNP) was unavailable for the outcome GWAS summary statistics, we identified proxy SNPs with a minimum linkage disequilibrium (LD) $r^{2}=0.8$. To minimize bias in effect estimates induced by correlation between SNPs, we restricted our genetic instrument to independent SNPs not in linkage disequilibrium $(p=0.0001)$. We refer to a set of SNPs that proxy sleep duration as "genetic instruments."

\section{Association of genetic instruments with outcome}

Genetic associations with renal function were obtained from the largest available extensively genotyped study based on a meta-analysis ( $n=133,413$ individuals with replication in up to 42,166 individuals) [22]. eGFR was estimated using the four-variable modification of diet in renal disease (MDRD) Study equation [22]. CKD was defined as eGFR $<60 \mathrm{ml} / \mathrm{min} / 1.73 \mathrm{~m}^{2}$. T2DM was defined as fasting glucose $\geq 126 \mathrm{mg} / \mathrm{dl}$, antidiabetic drug treatment or by self-reports. Kidney function and T2DM were assessed simultaneously.

For GWAS analysis, a centralized analysis plan was applied with each study regressing sex- and age-adjusted residuals of the logarithm of eGFR on SNP dosage levels. Furthermore, logistic regression of CKD was performed on SNP dosage levels adjusting for sex and age. For all traits, adjustment for appropriate study-specific features, such as study site and genetic principal components, was included 
Table 1 Summary results of the genetic loci of sleep duration obtained in varied methods

\begin{tabular}{|c|c|c|c|c|c|}
\hline SNP & GX & GX SE & EA & $\mathrm{OA}$ & EAF \\
\hline \multicolumn{6}{|l|}{ Self-reported } \\
\hline rs915416 & 0.019259 & 0.002495 & $\mathrm{C}$ & G & 0.289947 \\
\hline rs 269054 & -0.01364 & 0.002293 & $\mathrm{~T}$ & A & 0.577924 \\
\hline rs12567114 & -0.01483 & 0.00254 & $\mathrm{G}$ & $\mathrm{A}$ & 0.724198 \\
\hline rs62120041 & 0.026111 & 0.004575 & $\mathrm{~T}$ & $\mathrm{C}$ & 0.933902 \\
\hline rs374153 & 0.017612 & 0.003103 & $\mathrm{C}$ & $\mathrm{T}$ & 0.158085 \\
\hline rs75539574 & -0.03625 & 0.004065 & A & $\mathrm{C}$ & 0.914208 \\
\hline rs7556815 & -0.04072 & 0.00274 & G & A & 0.780856 \\
\hline rs12611523 & 0.012635 & 0.002276 & A & $\mathrm{G}$ & 0.545244 \\
\hline rs4538155 & -0.01298 & 0.002374 & $\mathrm{C}$ & $\mathrm{T}$ & 0.352574 \\
\hline rs10173260 & -0.01284 & 0.002313 & $\mathrm{~T}$ & $\mathrm{C}$ & 0.393765 \\
\hline rs112230981 & 0.031528 & 0.005228 & A & G & 0.94984 \\
\hline rs17732997 & 0.012935 & 0.002288 & $\mathrm{C}$ & G & 0.569098 \\
\hline rs7644809 & 0.013062 & 0.002301 & $\mathrm{~T}$ & $\mathrm{C}$ & 0.421606 \\
\hline rs13088093 & -0.01627 & 0.002402 & $\mathrm{~T}$ & G & 0.663683 \\
\hline rs2192528 & 0.013369 & 0.002269 & A & G & 0.480065 \\
\hline rs17427571 & 0.013826 & 0.002435 & A & G & 0.684313 \\
\hline rs35531607 & -0.01284 & 0.002273 & $\mathrm{~T}$ & $\mathrm{C}$ & 0.525917 \\
\hline rs13109404 & 0.031204 & 0.004408 & $\mathrm{~T}$ & G & 0.928024 \\
\hline rs 365663 & 0.014629 & 0.002279 & A & G & 0.545963 \\
\hline rs56372231 & -0.01694 & 0.0024 & $\mathrm{C}$ & $\mathrm{T}$ & 0.665907 \\
\hline rs 180769 & 0.012724 & 0.002294 & $\mathrm{~T}$ & $\mathrm{C}$ & 0.424698 \\
\hline rs151014368 & -0.01609 & 0.00282 & G & A & 0.793742 \\
\hline rs34556183 & 0.016923 & 0.002523 & A & $\mathrm{G}$ & 0.719606 \\
\hline rs80193650 & -0.01684 & 0.003067 & A & $\mathrm{G}$ & 0.837534 \\
\hline rs9382445 & 0.014536 & 0.002334 & $\mathrm{~T}$ & $\mathrm{C}$ & 0.62305 \\
\hline rs2231265 & -0.01496 & 0.002699 & A & G & 0.227711 \\
\hline rs34731055 & -0.01946 & 0.002948 & $\mathrm{C}$ & $\mathrm{T}$ & 0.81911 \\
\hline rs2079070 & 0.017548 & 0.002566 & $\mathrm{C}$ & G & 0.264613 \\
\hline rs7806045 & 0.014792 & 0.002626 & $\mathrm{~T}$ & $\mathrm{C}$ & 0.754703 \\
\hline rs330088 & -0.01447 & 0.002277 & $\mathrm{~T}$ & $\mathrm{C}$ & 0.452988 \\
\hline rs10973207 & -0.02043 & 0.003124 & G & $\mathrm{T}$ & 0.842323 \\
\hline rs1776776 & 0.019963 & 0.003411 & $\mathrm{~T}$ & $\mathrm{C}$ & 0.873832 \\
\hline rs12246842 & 0.013395 & 0.002274 & $\mathrm{~A}$ & $\mathrm{G}$ & 0.459815 \\
\hline rs10761674 & 0.012333 & 0.002266 & $\mathrm{C}$ & $\mathrm{T}$ & 0.477334 \\
\hline rs11190970 & 0.015379 & 0.002823 & G & A & 0.798661 \\
\hline rs7915425 & 0.019064 & 0.00299 & $\mathrm{~T}$ & $\mathrm{C}$ & 0.174682 \\
\hline rs1517572 & -0.01464 & 0.002295 & A & $\mathrm{C}$ & 0.419464 \\
\hline rs4592416 & -0.01468 & 0.00227 & A & G & 0.535593 \\
\hline rs 174560 & -0.01358 & 0.002437 & $\mathrm{~T}$ & $\mathrm{C}$ & 0.685785 \\
\hline rs12791153 & -0.02355 & 0.004217 & A & $\mathrm{T}$ & 0.918911 \\
\hline rs1939455 & 0.020425 & 0.003561 & G & $\mathrm{T}$ & 0.879446 \\
\hline rs1263056 & 0.012799 & 0.002277 & A & G & 0.519099 \\
\hline rs34354917 & 0.013746 & 0.002501 & $\mathrm{C}$ & A & 0.710472 \\
\hline rs11614986 & 0.016379 & 0.002951 & A & $\mathrm{G}$ & 0.820952 \\
\hline rs6575005 & 0.015564 & 0.002642 & $\mathrm{~T}$ & $\mathrm{C}$ & 0.757854 \\
\hline rs61985058 & -0.01859 & 0.003229 & $\mathrm{C}$ & $\mathrm{T}$ & 0.856824 \\
\hline rs11621908 & 0.024095 & 0.004163 & $\mathrm{C}$ & $\mathrm{T}$ & 0.917141 \\
\hline rs8038326 & 0.01592 & 0.002541 & A & $\mathrm{G}$ & 0.72691 \\
\hline
\end{tabular}

Table 1 (continued)

\begin{tabular}{lcclll}
\hline SNP & GX & GX SE & EA & OA & EAF \\
\hline rs3095508 & 0.015352 & 0.002304 & C & A & 0.593529 \\
rs11643715 & -0.0139 & 0.002497 & C & G & 0.709058 \\
rs9940646 & 0.016946 & 0.002291 & C & G & 0.577569 \\
rs7503199 & 0.014745 & 0.002564 & C & T & 0.734267 \\
rs1991556 & 0.016566 & 0.002724 & G & A & 0.773765 \\
rs12607679 & 0.020139 & 0.002593 & T & C & 0.737717 \\
rs10421649 & -0.0133 & 0.002295 & T & A & 0.44303 \\
rs2072727 & 0.013243 & 0.002285 & T & C & 0.43617 \\
Accelerometer derived & & & & & \\
rs2660302 & 0.041 & 0.006 & A & T & 0.811 \\
rs113851554 & 0.11 & 0.011 & G & T & 0.943 \\
rs62158170 & 0.054 & 0.006 & G & A & 0.217 \\
rs17400325 & 0.066 & 0.012 & T & C & 0.958 \\
rs72828540 & 0.041 & 0.005 & T & C & 0.752 \\
rs9369062 & 0.033 & 0.005 & C & A & 0.292 \\
rs2975734 & 0.027 & 0.005 & C & G & 0.561 \\
rs13282541 & 0.032 & 0.005 & C & T & 0.739 \\
rs2880370 & 0.028 & 0.005 & A & T & 0.67 \\
rs800165 & 0.028 & 0.005 & T & T & 0.343 \\
rs10138240 & 0.029 & 0.005 & G & G & 0.514 \\
\hline
\end{tabular}

$E A$ effect allele, $O A$ other allele, $E A F$ effect allele frequency, $G X$ the per-allele effect on standard deviation units of the telomere length, $G X S E$ standard error of GX

in the regression and family-based studies appropriately accounted for relatedness.

\section{MR analysis}

We combined the effect of five instruments using inverse variance weighted (IVW) method as implemented in the two sample MR package running under $\mathrm{R}$ [version 3.4.2 $\mathrm{R}$ Core Team (2017)]. We assessed the heterogeneity using $Q$ value for IVW. To address potential effect of pleiotropic variants on the final effect estimate, we performed a sensitivity analysis including weighted median (WM) and MR-Egger. Sensitivity analysis was conducted using the leave-one-out method to identify instruments that might drive the MR results. The WM estimate provides correct estimates as long as SNPs accounting for $\geq 50 \%$ of the weight are valid instruments. Inverse variance is used to weight the variants and bootstrapping is applied to estimate the confidence intervals (CIs) [23]. MR-Egger has an ability to make estimates even under the assumption that all SNPs are invalid instruments, as long as the assumption of instrument strength independent of direct effect (InSIDE) is satisfied [23]. However, the InSIDE assumption cannot be easily verified. Average directional pleiotropy across genetic variants was assessed from the $\mathrm{p}$ value of the intercept term from MR-Egger [23]. Causal 
estimates in MR-Egger are less precise than those obtained by IVW MR [24]. Analysis using MR-Egger has a lower false-positive rate but a higher false-negative rate than IVW, i.e. it has a lower statistical power [25].

To assess heterogeneity between individual genetic variant estimates, we performed the $\mathrm{Q}^{\prime}$ heterogeneity statistic [26] and the MR pleiotropy residual sum and outlier (MRPRESSO) test [26]. The $\mathrm{Q}^{\prime}$ statistic uses modified secondorder weights that are a derivation of a Taylor series expansion and consider the uncertainty in both numerator and denominator of the instrumental variable ratio [26]. The MR-PRESSO framework detects effect estimates that are outliers and removes them from the analysis. This is done by regressing the variant-outcome associations on variantexposure associations. A global heterogeneity test is then implemented, comparing the observed distance between residual sums of squares of all variants to the regression line with the distance expected under the null hypothesis of no pleiotropy [27]. Furthermore, we applied on MRRobust Adjusted Profile Score (RAPS); this method is able to correct for pleiotropy using robust adjusted profile scores. RAPS can provide an unbiased causal estimate in the presence of weak instruments. We consider as results causal estimates that agreed in direction and magnitude across MR methods, passed nominal significance in IVW MR and did not show any evidence of bias from horizontal pleiotropy using heterogeneity tests. To assess the instrumental variable analysis "exclusion-restriction" assumption, we used
Ensembl release (https://useast.ensembl.org/index.html) that contains a base of SNP phenotypes.

\section{Ethics}

This investigation uses published or publicly available summary data with no further involvement of participants. No original data were collected for this study. Ethical approval for each of the studies included in the analyses can be found in the original publications (including informed consent from each participant). The study conforms to the ethical guidelines of the 1975 Declaration of Helsinki.

\section{Results}

\section{Mendelian randomization (MR) analysis}

The list of all instruments' associations for sleep duration (subjective and objective) is shown in Table 1. The results, expressed as $\beta$-coefficient for sleep duration per 1 standard deviation (SD) increase in outcomes, are presented in Table 2 (for self-reported sleep duration) and Table 3 (for accelerometer-derived sleep duration). With regard to the self-reported sleep duration, individuals with longer sleep duration had a greater risk of CKD (IVW: $\beta=0.358, p=0.047$ ) in the total population. A significant link between longer self-reported sleep duration and lower eGFR was also observed but only

Table 2 Results of the Mendelian randomization (MR) analysis for all exposures (for self-reported sleep duration)

\begin{tabular}{|c|c|c|c|c|c|c|c|c|c|c|}
\hline \multirow[t]{2}{*}{ Exposures } & \multicolumn{4}{|l|}{ MR } & \multicolumn{3}{|c|}{ Heterogeneity } & \multicolumn{3}{|l|}{ Pleiotropy } \\
\hline & Method & Beta & SE & $p$ & Method & $Q$ & $p$ value & Intercept & SE & $p$ \\
\hline \multirow[t]{4}{*}{ CKD } & MR-Egger & 0.9034 & 0.6866 & 0.1966 & MR-Egger & 45.34 & 0.1368 & -0.0093 & 0.011 & 0.415 \\
\hline & WM & 0.4764 & 0.2685 & 0.07603 & & & & & & \\
\hline & IVW & 0.3583 & 0.1856 & 0.047 & IVW & 46.2 & 0.1428 & & & \\
\hline & RAPS & 0.4413 & 0.1941 & 0.02299 & & & & & & \\
\hline \multicolumn{11}{|l|}{ eGFR } \\
\hline \multirow[t]{4}{*}{ Totali } & MR-Egger & -0.0409 & 0.03856 & 0.2959 & MR-Egger & 45.05 & 0.1434 & 0.00038 & 0.00064 & 0.562 \\
\hline & WM & -0.01982 & 0.01604 & 0.2165 & & & & & & \\
\hline & IVW & -0.01913 & 0.01066 & 0.0727 & IVW & 45.48 & 0.1597 & & & \\
\hline & RAPS & -0.02149 & 0.01091 & 0.04876 & & & & & & \\
\hline \multirow[t]{4}{*}{ Non-DM } & MR-Egger & -0.06524 & 0.04006 & 0.1121 & MR-Egger & 42.11 & 0.2235 & 0.00069 & 0.00066 & 0.302 \\
\hline & WM & -0.03157 & 0.01585 & 0.04633 & & & & & & \\
\hline & IVW & -0.02476 & 0.01066 & 0.02016 & IVW & 43.39 & 0.2175 & & & \\
\hline & RAPS & -0.02577 & 0.01084 & 0.01744 & & & & & & \\
\hline \multirow[t]{4}{*}{$\mathrm{DM}$} & MR-Egger & 0.04857 & 0.1499 & 0.7478 & MR-Egger & 37.96 & 0.3801 & -0.00035 & 0.0025 & 0.888 \\
\hline & WM & 0.02369 & 0.06189 & 0.7019 & & & & & & \\
\hline & IVW & 0.02804 & 0.04007 & 0.4841 & IVW & 37.98 & 0.4245 & & & \\
\hline & RAPS & 0.02957 & 0.04148 & 0.4759 & & & & & & \\
\hline
\end{tabular}

$W M$ weighted median, $I V W$ inverse variance weighted, $S E$ standard error, beta beta-coefficients, $M R$ Mendelian randomization, $C K D$ chronic kidney disease, $e G F R$ estimated glomerular filtration rate, $T 2 D M$ type 2 diabetes mellitus 
Table 3 Results of the mendelian randomization (MR) analysis for all exposures (for accelerometer derived sleep duration)

\begin{tabular}{|c|c|c|c|c|c|c|c|c|c|c|}
\hline \multirow[t]{2}{*}{ Exposures } & \multicolumn{4}{|l|}{ MR } & \multicolumn{3}{|c|}{ Heterogeneity } & \multicolumn{3}{|l|}{ Pleiotropy } \\
\hline & Method & Beta & SE & $p$ & Method & $Q$ & $p$ value & Intercept & SE & $p$ \\
\hline \multirow[t]{4}{*}{ CKD } & MR-Egger & 1.557 & 0.7086 & 0.07938 & MR-Egger & 2.765 & 0.7362 & -0.066 & 0.028 & 0.0665 \\
\hline & WM & 0.1731 & 0.2271 & 0.4459 & & & & & & \\
\hline & IVW & -0.04924 & 0.204 & 0.8093 & IVW & 8.232 & 0.2216 & & & \\
\hline & RAPS & -0.01708 & 0.2092 & 0.9349 & & & & & & \\
\hline \multicolumn{11}{|l|}{ eGFR } \\
\hline \multirow[t]{4}{*}{ Total } & MR-Egger & -0.03524 & 0.04022 & 0.421 & MR-Egger & 1.4 & 0.9243 & 0.00065 & 0.0016 & 0.706 \\
\hline & WM & -0.01553 & 0.01165 & 0.1824 & & & & & & \\
\hline & IVW & -0.01969 & 0.01 & 0.04702 & IVW & 1.559 & 0.9555 & & & \\
\hline & RAPS & -0.01978 & 0.01038 & 0.05679 & & & & & & \\
\hline \multirow[t]{4}{*}{ Non-DM } & MR-Egger & -0.03565 & 0.04178 & 0.4325 & MR-Egger & 2.015 & 0.847 & 0.00043 & 0.0017 & 0.808 \\
\hline & WM & -0.02424 & 0.01263 & 0.055 & & & & & & \\
\hline & IVW & -0.02527 & 0.01038 & 0.01486 & IVW & 2.081 & 0.9121 & & & \\
\hline & RAPS & -0.02541 & 0.01082 & 0.01891 & & & & & & \\
\hline \multirow[t]{4}{*}{$\mathrm{DM}$} & MR Egger & -0.2975 & 0.1684 & 0.1377 & MR-Egger & 4.758 & 0.4461 & 0.011 & 0.0068 & 0.153 \\
\hline & WM & -0.01371 & 0.05743 & 0.8113 & & & & & & \\
\hline & IVW & -0.02287 & 0.04688 & 0.6256 & IVW & 7.589 & 0.2698 & & & \\
\hline & RAPS & -0.02561 & 0.04763 & 0.5908 & & & & & & \\
\hline
\end{tabular}

$W M$ weighted median, $I V W$ inverse variance weighted, $S E$ standard error, beta beta-coefficients, $M R$ mendelian randomization, $C K D$ chronic kidney disease, $e G F R$ estimated glomerular filtration rate, $D M$ diabetes

among non-diabetics (IVW: $\beta=-0.024, p=0.020$ ). No significant association was found between self-reported sleep duration and eGFR in the whole population (IVW: $\beta=-0.019$, $p=0.072$ ) and T2DM patients (IVW: $\beta=0.028, p=0.484$ ).

With regard to the accelerometer-derived sleep duration, individuals with longer sleep duration had a lower eGFR both in the total population (IVW: $\beta=-0.019, p=0.047$ ) and the non-diabetics (IVW: $\beta=-0.025, p=0.014$ ), but not among T2DM patients (IVW: $\beta=-0.022, p=0.625$ ). Furthermore, there was no significant link between sleep duration and the risk for CKD in the total population, the non-diabetics and the T2DM group.

None of the IWV estimates showed heterogeneity. MRPRESSO analysis did not show any possibility of outlier for all of the estimates. The pleiotropy test, with very negligible intercept and insignificant $\mathrm{p}$ value, also indicated low chance of the pleiotropy for all of our estimations (all $p>0.539$ ). The results of the MR-RAPS were identical with the IVW estimates, highlighting again a low likelihood of pleiotropy. The results of the leave-one-out method demonstrated that the links were not driven by single SNPs.

\section{Discussion}

In the present study, we performed a MR analysis to evaluate the impact of sleep durationon the risk of CKD and renal function indexes in a casual mode. Potential harmful effects of longer sleep duration (measured both objectively and subjectively) on kidney function were found. This finding was observed in the total population and in non-diabetic individuals, but not in those with T2DM, with low level of the heterogeneity and chance of pleiotropic.

There are several plausible mechanisms regarding the association of sleep duration with renal function that need to be further elucidated. Most renal physiological processes follow a circadian rhythm, including the regulation of sodium excretion, renin-angiotensin system and blood pressure [28]. Therefore, disruption in sleeping patterns (short or long sleep duration) may negatively affect this chronobiological process and lead to renal dysfunction. In this context, disruption in cyclic behavioural patterns in animal models was associated with proteinuria, glomerulosclerosis, tubular hyperplasia, and renal fibrosis [29]. Furthermore, sleeping $5 \mathrm{~h}$ or fewer per night was more related to the development of hypertension and T2DM during a 10-year follow-up period compared with sleeping $7 \mathrm{~h}$ per night $[5,30]$. In short term studies, lasting 3-6 weeks, sleep duration was a modifiable predictor of insulin resistance and blood pressure [31, 32]. Short sleep duration was associated with a rapid decline in renal function independently of established CKD risk factors such as blood pressure and insulin resistance [11]. Moreover, sleep duration may affect the levels of pro-inflammatory markers such as interleukin-6 and C-reactive protein $[33,34]$ that play an important role in CKD pathogenesis [35]. Obstructive sleep apnea (OSA) 
has been linked to the risk of CKD [36, 37] via enhanced sympathetic activation, oxidative and nitrosative stress, and impaired microvascular and endothelial function in T2DM patients, leading to renal dysfunction $[38,39]$. Taking into consideration the association between sleep duration and OSA [40], sleep duration may also affect the risk for CKD development via this pathway.

A few studies investigated the relationship between sleep duration and CKD with controversial results. Cross-sectional studies have previously reported that advanced CKD can lead to a higher prevalence of several sleep disorders, including reversal of day-night sleep patterns, increased sleep latency, and fragmented sleep related to sleep apnea or restless leg syndrome [9]. In this context, shorter sleep duration (i.e. $<5 \mathrm{~h}$ ) measured by actigraphy was significantly more prevalent in non-dialysis dependent CKD and end-stage renal disease (ESRD) patients compared with individuals with normal kidney function [41]. Ohkuma et al. [14] reported that both short and long sleep duration were associated with higher UACR (urinary albumin-tocreatinine ratio) and albuminuria. Moreover, consistent with our observations, lower eGFR correlated with long sleep duration independently of potential confounders [14]. In a population-based cross-sectional study of $1258 \mathrm{~T} 2 \mathrm{DM}$ patients (aged $40-80$ years), long sleep duration ( $>8 \mathrm{~h}$ ) was associated with renal insufficiency compared with normal sleep duration (7-8 h) [13]. In the same study, both long and very short duration of sleep $(<5 \mathrm{~h})$ were linked with albuminuria; after adjusting for potential confounders, only long sleep duration remained significantly related to 2.3fold higher odds of low eGFR [13]. Another study of 6834 Japanese adults (aged 20-65 years) reported that proteinuria was more frequently developed in individuals who slept 6 or fewer hours than those who slept for 7-8 h per night; however, eGFR did not differ between these individuals [12]. Similarly, sleep parameters were not associated with a 5-year change in eGFR in a prospective study of 463 adults (aged 32-51 years) [42]. However, each $1 \mathrm{~h}$ decrease in sleep duration was significantly related to a $1.5 \mathrm{ml} / \mathrm{min} / 1.73 \mathrm{~m}^{2}$ higher eGFR [42].

In contrast to our results, a systematic review and metaanalysis of 6 observational studies with 252,075 individuals and 3 observational studies with 37,197 participants reported a positive significant association between short sleep duration and proteinuria, but no association between short sleep duration and CKD [10]. In another study, short (but not long) sleep duration was related to diabetic CKD (DKD), defined as the presence of albuminuria and eGFR $<60 \mathrm{ml} / \mathrm{min} / 1.73$ $\mathrm{m}^{2}$ [15]. Furthermore, a prospective cohort study of 4238 individuals from the Nurse Health Study over an 11-year period reported that shorter sleep duration was associated with a rapid reduction in eGFR [11]. Of note, women who slept $5 \mathrm{~h}$ or less per night experienced the quickest decline in renal function, whereas the slowest rate of decline was observed in those who reported sleeping 7-8 h per night [11]. Moreover, albuminuria was twice as prevalent among those who slept 5 or fewer hours per night compared with those who reported 7-8 h of sleep per night [11]. However, some limitations should be considered for this study. First of all, sleep duration was collected 3 years prior to initial eGFR measurement. Second, the U-shaped association between sleep duration and rapid decline in renal function was not assessed due to the limited number of individuals reporting sleep duration of $9 \mathrm{~h}$ or more [11]. Third, the study population was limited to women, and most individuals were white. Therefore, findings cannot be applied to men and other racial groups.

The present study has several strengths, e.g. the possibility of reverse causation was minimized, genotypes were assumed to be randomly distributed with respect to confounders, the ability to perform an array of sensitivity analyses was enhanced by the use of pleiotropy-robust methods. There are also certain limitations, including the inability to thoroughly evaluate individual-level confounding factors and horizontal pleiotropy.

In conclusion, potential harmful effects of longer sleep duration (measured both objectively and subjectively) on kidney function was observed in a casual mode in the present MR analysis. This finding was observed both in total population and non-diabetic individuals, but not in those with T2DM. Further research is needed in this field to elucidate the associations between sleep duration and renal function.

Acknowledgements The material presented in this manuscript have not been published previously in whole or part, except in abstract format.

Funding This research did not receive any specific grant from funding agencies in the public, commercial, or non-for-profit sectors.

\section{Compliance with ethical standards}

Conflict of interest All authors declare that they have no conflict of interest.

Open Access This article is licensed under a Creative Commons Attribution 4.0 International License, which permits use, sharing, adaptation, distribution and reproduction in any medium or format, as long as you give appropriate credit to the original author(s) and the source, provide a link to the Creative Commons licence, and indicate if changes were made. The images or other third party material in this article are included in the article's Creative Commons licence, unless indicated otherwise in a credit line to the material. If material is not included in the article's Creative Commons licence and your intended use is not permitted by statutory regulation or exceeds the permitted use, you will need to obtain permission directly from the copyright holder. To view a copy of this licence, visit http://creativecommons.org/licenses/by/4.0/. 


\section{References}

1. Sharma S, Kavuru M (2010) Sleep and metabolism: an overview. Int J Endocrinol 2010

2. Kripke DF, Garfinkel L, Wingard DL, Klauber MR, Marler MR (2002) Mortality associated with sleep duration and insomnia. Arch Gen Psychiatry 59(2):131-136

3. Ferrie JE, Shipley MJ, Cappuccio FP et al (2007) A prospective study of change in sleep duration: associations with mortality in the Whitehall II cohort. Sleep 30(12):1659-1666

4. Ayas NT, White DP, Manson JE et al (2003) A prospective study of sleep duration and coronary heart disease in women. Arch Intern Med 163(2):205-209

5. Ayas NT, White DP, Al-Delaimy WK et al (2003) A prospective study of self-reported sleep duration and incident diabetes in women. Diabetes Care 26(2):380-384

6. Cappuccio FP, Stranges S, Kandala N-B et al (2007) Genderspecific associations of short sleep duration with prevalent and incident hypertension: the Whitehall II Study. Hypertension 50(4):693-700

7. Gangwisch JE, Heymsfield SB, Boden-Albala B et al (2006) Short sleep duration as a risk factor for hypertension: analyses of the first National Health and Nutrition Examination Survey. Hypertension 47(5):833-839

8. Yaggi HK, Araujo AB, McKinlay JB (2006) Sleep duration as a risk factor for the development of type 2 diabetes. Diabetes Care 29(3):657-661

9. Turek NF, Ricardo AC, Lash JP (2012) Sleep disturbances as nontraditional risk factors for development and progression of CKD: review of the evidence. Am J Kidney Dis 60(5):823-833

10. Cheungpasitporn W, Thongprayoon C, Gonzalez-Suarez ML et al (2016) The effects of short sleep duration on proteinuria and chronic kidney disease: a systematic review and meta-analysis. Nephrol Dial Transpl 32(6):991-996

11. McMullan CJ, Curhan GC, Forman JP (2016) Association of short sleep duration and rapid decline in renal function. Kidney Int 89(6):1324-1330

12. Yamamoto R, Nagasawa Y, Iwatani H et al (2012) Self-reported sleep duration and prediction of proteinuria: a retrospective cohort study. Am J Kidney Dis 59(3):343-355

13. Tan N, Chan J, Cheng C-Y, Wong TY, Sabanayagam C (2018) Sleep duration and diabetic kidney disease. Front Endocrinol 9:808

14. Ohkuma T, Fujii H, Iwase M et al (2013) Association between sleep duration and urinary albumin excretion in patients with type 2 diabetes: the Fukuoka diabetes registry. PLoS ONE 8(11):e78968

15. Meng L-L, Liu Y, Geng R-N, Tang Y-Z, Li D-Q (2016) Association of diabetic vascular complications with poor sleep complaints. Diabetol Metab Syndr 8(1):80

16. Zuber AM, Centeno G, Pradervand S et al (2009) Molecular clock is involved in predictive circadian adjustment of renal function. Proc Natl Acad Sci 106(38):16523-16528

17. Davies NM, Holmes MV, Davey SG (2018) Reading Mendelian randomisation studies: a guide, glossary, and checklist for clinicians. BMJ 362:k601

18. Smith GD, Ebrahim S (2003) 'Mendelian randomization': can genetic epidemiology contribute to understanding environmental determinants of disease? Int J Epidemiol 32(1):1-22

19. Bycroft C, Freeman C, Petkova D et al (2018) The UK Biobank resource with deep phenotyping and genomic data. Nature 562(7726):203-209

20. Dashti HS, Jones SE, Wood AR et al (2019) Genome-wide association study identifies genetic loci for self-reported habitual sleep duration supported by accelerometer-derived estimates. Nat Commun 10(1):1100

21. Jones SE, van Hees VT, Mazzotti DR et al (2019) Genetic studies of accelerometer-based sleep measures yield new insights into human sleep behaviour. Nat Commun 10(1):1585

22. Pattaro C, Teumer A, Gorski M et al (2016) Genetic associations at 53 loci highlight cell types and biological pathways relevant for kidney function. Nat Commun 7:10023

23. Bowden J, Davey Smith G, Haycock PC, Burgess S (2016) Consistent estimation in mendelian randomization with some invalid instruments using a weighted median estimator. Genet Epidemiol 40(4):304-314

24. Bowden J, Davey Smith G, Burgess S (2015) Mendelian randomization with invalid instruments: effect estimation and bias detection through Egger regression. Int J Epidemiol 44(2):512-525

25. Burgess S, Bowden J, Fall T, Ingelsson E, Thompson SG (2017) Sensitivity analyses for robust causal inference from Mendelian randomization analyses with multiple genetic variants. Epidemiology 28(1):30-42

26. Bowden J, Del Greco MF, Minelli C, Davey Smith G, Sheehan N, Thompson J (2017) A framework for the investigation of pleiotropy in two-sample summary data Mendelian randomization. Stat Med 36(11):1783-1802

27. Verbanck M, Chen CY, Neale B, Do R (2018) Detection of widespread horizontal pleiotropy in causal relationships inferred from Mendelian randomization between complex traits and diseases. Nat Genet 50(5):693-698

28. Solocinski K, Gumz ML (2015) The circadian clock in the regulation of renal rhythms. J Biol Rhythms 30(6):470-486

29. Martino TA, Oudit GY, Herzenberg AM et al (2008) Circadian rhythm disorganization produces profound cardiovascular and renal disease in hamsters. Am J Physiol Regul Integr Comp Physiol 294(5):R1675-R1683

30. Gangwisch JE, Feskanich D, Malaspina D, Shen S, Forman JP (2013) Sleep duration and risk for hypertension in women: results from the nurses' health study. Am J Hypertens 26(7):903-911

31. Haack M, Serrador J, Cohen D, Simpson N, Meier-Ewert H, Mullington JM (2013) Increasing sleep duration to lower beat-to-beat blood pressure: a pilot study. J Sleep Res 22(3):295-304

32. Buxton OM, Cain SW, O'Connor SP et al (2012) Adverse metabolic consequences in humans of prolonged sleep restriction combined with circadian disruption. Sci Transl Med 4(129):129ra143-129ra143

33. Grandner MA, Sands-Lincoln MR, Pak VM, Garland SN (2013) Sleep duration, cardiovascular disease, and proinflammatory biomarkers. Nat Sci Sleep 5:93

34. Miller MA, Kandala N-B, Kivimaki M et al (2009) Gender differences in the cross-sectional relationships between sleep duration and markers of inflammation: Whitehall II study. Sleep 32(7):857-864

35. Choudhary N, Ahlawat RS (2008) Interleukin-6 and C-Reactive protein in pathogenesis of diabetic nephropathy new evidence linking inflammation, glycemic control, and microalbuminuria. Iran J Kidney Dis 2(2):72-79

36. Chou Y-T, Lee P-H, Yang C-T et al (2011) Obstructive sleep apnea: a stand-alone risk factor for chronic kidney disease. Nephrol Dial Transpl 26(7):2244-2250

37. Tahrani AA, Ali A, Raymond NT et al (2013) Obstructive sleep apnea and diabetic nephropathy: a cohort study. Diabetes Care 36(11):3718-3725

38. Nácher M, Farré R, Montserrat JM et al (2009) Biological consequences of oxygen desaturation and respiratory effort in an acute animal model of obstructive sleep apnea (OSA). Sleep Med 10(8):892-897 
39. He Q, Yang Q-c, Zhou Q et al (2014) Effects of varying degrees of intermittent hypoxia on proinflammatory cytokines and adipokines in rats and 3T3-L1 adipocytes. PLoS One 9(1):e86326

40. Flemons WW (2002) Obstructive sleep apnea. N Engl J Med 347(7):498-504

41. Agarwal R, Light RP (2011) Sleep and activity in chronic kidney disease: a longitudinal study. Clin J Am Soc Nephrol 6(6): 1258-1265

42. Petrov ME, Kim Y, Lauderdale DS et al (2014) Objective sleep, a novel risk factor for alterations in kidney function: the CARDIA study. Sleep Med 15(9):1140-1146
Publisher's Note Springer Nature remains neutral with regard to jurisdictional claims in published maps and institutional affiliations. 